\title{
The Application of Image Segmentation to Determine the Ratio of Peritumoral Edema Area to Tumor Area on Primary Malignant Brain Tumor and Metastases through Conventional Magnetic Resonance Imaging
}

\author{
Bestia Kumala Wardani ${ }^{1}$, Yuyun Yueniwati ${ }^{2 *}$ (D) Agus Naba $^{3}$ D \\ ${ }^{1}$ Biomedical Science Study Program, Faculty of Medicine, Universitas Brawijaya, Malang, Indonesia; ${ }^{2}$ Department of Radiology, \\ Faculty of Medicine, Universitas Brawijaya, Saiful Anwar Hospital, Malang, Indonesia; ${ }^{3}$ Department of Physics, Faculty of \\ Mathematics and Natural Sciences, Universitas Brawijaya, Malang, Indonesia
}

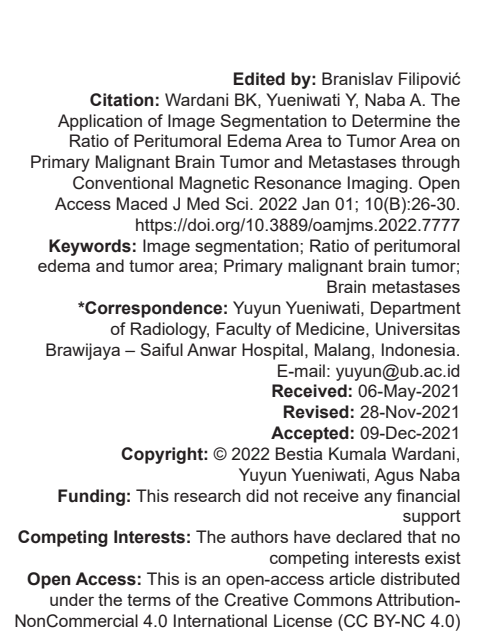

Abstract

BACKGROUND: Primary malignant brain tumor and metastases on the brain have a similar pattern in conventional magnetic resonance imaging (MRI), even though both require entirely different treatment and management. The pathophysiological difference of peritumoral edema can help to distinguish the case of primary malignant brain tumor and brain metastases.

AIM: This study aimed to analyze the ratio of the area of peritumoral edema to the tumor using Otsu's method of image segmentation technique with a user-friendly graphical user interface.

METHODS: Data were prepared by obtaining the examination results of anatomical pathology and MRI imaging. The area of peritumoral edema was identified from MRI image segmentation with T2/fluid attenuated inversion recovery sequence. The area of tumor was identified using MRI image segmentation with T1 sequence.

RESULTS: The Mann-Whitney test was employed to analyze the ratio of the area of peritumoral edema to tumor on both groups. Data testing produced a significance level of $0.013(p<0.05)$ with a median value $\left(\mathrm{N}_{\max }-\mathrm{N}_{\min }\right)$ of $1.14(3.31-0.08)$ for the primary malignant brain tumor group and a median value $\left(\mathrm{N}_{\max }-\mathrm{N}_{\min }\right)$ of $1.17(10.30-0.90)$ for the brain metastases group.

CONCLUSIONS: There was a significant difference in the ratio of the area of peritumoral edema to the area of tumor from both groups, in which brain metastases have a greater value than the primary malignant brain tumor.

\section{Introduction}

A brain tumor is the collection of tissue mass in the brain as a result of abnormal and uncontrolled cell growth. Clinically, brain tumor can be divided into two categories, which are primary brain tumor and secondary brain tumor (metastases) [1]. Peritumoral edema can be one indicator of tumor malignancy in evaluating radiological images [2]. In primary malignant brain tumors, peritumoral edema is often referred to as "infiltrative edema" because it is vasogenic edema infiltrated by tumor cells. In brain metastases, peritumoral edema essentially consists of pure vasogenic edema because due to not containing any tumor cell infiltrates [3].

Non-invasive magnetic resonance imaging (MRI) technology can curtail surgical procedures, which often lead to an increased risk of death [4]. Despite the various improvements in advanced imaging techniques in the past decades, the use of conventional MRI remains the most frequently practiced standard method of treatment imaging [5]. Its ability is deeply limited, which oftentimes causes confusion in distinguishing the development of the two different types of brain tumor. Especially in cases of a solitary lesion, primary malignant brain tumor and brain metastases have a near-identical pattern in MRI imaging, despite the entirely different treatment and management. Patients with primary malignant brain tumors will immediately undergo surgical resection, whereas those with brain metastases will have to first go through a more intricate identification process to determine cancer's site of origin before deciding the subsequent therapy procedures. Lengthy and inaccurate diagnoses will further aggravate the patient's condition [6]. Two sequences of conventional MRI that can be used to observe the aforementioned comparison are the fluid attenuated inversion recovery (FLAIR) sequence for the visualization of peritumoral edema and the T1W1 
sequence with contrast to allow for the visualization of tumor.

Conventional MRI does not yet have designated software to automatically distinguish primary malignant brain tumor from brain metastases. Assessment is still undertaken manually, through direct reading by the radiologist, which is subjective in nature because it relies on the capability of each individual. Manual reading also tends to require a longer time and cause a heavier workload, all of which may allow the possibility of image interpretation errors [7]. A new strategy is needed to enhance conventional MRI's ability to tell primary malignant brain tumors apart from brain metastases. Accurate establishment of diagnosis will help a radiologist in determining an immediate and precise treatment plan [8].

Imaging data can be quantified and developed into a strong biomarker that improves clinical decision-making as a problem-solving means in treating patients [9]. An analysis of the shape and size of images calls for accurate and precise delineation of lesion margins for the segmentation process, which will later be used as the standard for calculation [10]. This study was conducted by analyzing the ratio of the area of peritumoral edema to the area of tumor on the conventional MRI images using Otsu's method of image segmentation technique. Image segmentation is a process of partitioning digital images into several segments or groups, which are a collection of pixels. It divided the images into discrete regions. Thus, pixels have a high similarity level in each region and represent each region [11]. Thresholding is a type of segmentation method that serves to isolate images based on the cutoff value. One of the methods to obtain threshold value is through Otsu's method. This is a popular and the best method among all of the thresholding methods in automatically obtaining the threshold value [10]. A more effective and efficient technology can improve the quality of life of patients with these two types of brain tumors [12, 13].

\section{Methods}

\section{Study sample and population}

The population of this study is patients with a primary malignant brain tumor and brain metastases who have their results from anatomical pathology assessment and MRI. The samples used in this study were $33 \mathrm{MRI}$ images, ten of which were of primary malignant brain tumor and the remaining 23 were of brain metastases. This study has fulfilled both the inclusion and exclusion criteria. The inclusion criteria were patients with primary malignant brain tumor who have their MRI results and pathological examination result. Meanwhile, the exclusion criteria consisted of patients with a brain tumor who have their MRI results, but not their pathological examination results; patients with a primary malignant brain tumor with extraaxial location or outside the brain parenchyma; and secondary brain tumors resulting from direct invasion from the primary tumor [14].

\section{Study method}

This study commenced with the design of graphical user interface (GUI), in which lies the arrays of written programming codes that represent an instruction of digital image processing. Digital image processing is a technology that applies several computer algorithms to process digital images [15]. Through the FLAIR sequence, this study was able to segment the peritumoral edema and tumor regions. This region was symbolized as the region "a." Meanwhile, through the T1 sequence with contrast, the tumor region was able to be segmented. This region was symbolized as region "b." The area of the segmentation result of regions "a" and "b" can then be calculated. The ratio of the area of peritumoral edema to the area of tumor was calculated using the formula: $(a-b) / b$.

\section{Statistical analysis}

Because one of the data did not have normal distribution as required for the parametric test, then the ratio of the area of peritumoral edema to the area of tumor from the MRI images was tested using the nonparametric Mann-Whitney statistical test.

\section{Results}

Sampling was done from June 2020 to August 2020 with a total of 33 samples.

The number of samples in this study was 33 , consisting of ten primary malignant brain tumor samples and 23 brain metastases samples. The most of the samples were female patients with a proportion of $69.7 \%$, with the remaining $30.3 \%$ male patients (Table 1 ).

The differences in the measurement result between the patient groups of primary malignant brain tumor and brain metastases were able to be identified through the non-parametric Mann-Whitney statistical test. Ratio data of the area of peritumoral edema to the area of tumor in the primary malignant brain tumor group and the brain metastases group produced a significance level of $0.013(p<0.05)$, which means that there is a significant difference regarding the ratio of the area of peritumoral edema to the area of tumor in the patient groups with a primary malignant brain tumor and brain 
Table 1: The characteristics of the number of tumors based on sex

\begin{tabular}{llll}
\hline Sex & \multicolumn{2}{l}{ The number of tumors } & Total \\
\cline { 2 - 3 } & Primary malignant & Metastasis & \\
\hline Male $(\%)$ & $4(12.1)$ & $6(18.2)$ & $10(30.3)$ \\
Female $(\%)$ & $6(18.2)$ & $17(51.5)$ & $23(69.7)$ \\
Total & & & $33(100)$ \\
\hline
\end{tabular}

metastases. Based on the test results of the ratio data between the area of peritumoral edema and the area of brain metastases, this study discovered that the primary malignant brain tumor group had a median value $\left(\mathrm{N}_{\max }{ }^{-}\right.$ $\mathrm{N}_{\min }$ ) of 1.14 (3.31-0.08) and the brain metastases group had a median value of $\left(\mathrm{N}_{\max }-\mathrm{N}_{\min }\right)$ of $1.17(10.30-0.90)$ (Table 2). There was a significant difference in the ratio of the area of peritumoral edema to the area of tumor from both groups, in which brain metastases have a greater value than the malignant brain tumor.

Table 2: A comparison data between primary malignant brain tumor and brain metastases

\begin{tabular}{lll}
\hline Variable & \multicolumn{2}{l}{ Median (Max-Min) } \\
\cline { 2 - 3 } & Primary malignant brain tumor & Brain metastatic tumor \\
\hline Area of tumor (a) & $2376.69(5293.00-211.87)$ & $898.12(4619.75-123.87)$ \\
Area of edema (b) & $3018.95(4595.72-183.25)$ & $2771.83(6075.08-236.70)$ \\
Ratio (b/a) & $1.14(3.31-0.08)$ & $1.17(10.30-0.90)$ \\
\hline
\end{tabular}

GUI acts as the graphical display that will appear when the user operates the program. With a GUI, the program is made to be more users friendly so that it is easy for users to run an application program. GUI can be regarded as optimal when the program becomes easier to be used by providing simple display or interface features, such as push-button, list boxes, sliders, menus, and so on. Commencing a process by opening the data repository of brain tumor images through clicking the "open file" push-button, and the images that have been chosen will be displayed automatically on the panels
"FLAIR" and "T1." Next, to level the images' size, the user can select the "rp" or "rpp" push-buttons. The crop push button function is to crop and focus the parts that contain the tumor and edema. The slider command can be moved sideways to determine the threshold value, which then can produce a binary image of black and white. The image segmentation aims to identify the objects contained within the image, that is, edema and tumor, which will then be separated from their background. The desired object is adjusted to be colored white (pixel intensity value is one), while the rest are black (pixel intensity value is zero). The segmentation of brighter regions (white) can be selected using the button "FLAIR w" and "T1 w." Meanwhile, the segmentation of darker regions (black) can be selected using the button "FLAIR b" and "T1 b." The "FLAIR 2" area here is the combination of the area of edema and the area of the tumor, while the "T1 2" area is the area of the tumor. The area of FLAIR and T1 will be automatically calculated. The area of edema can be obtained from the subtraction of the FLAIR area with the T1 area. The area of tumor can be found from the area of T1. The "ratio" button will automatically compute the comparison value (ratio) between the area of edema and the area of the tumor. The "clear" button will restart the calculation for new images (Figure 1).

\section{Discussion}

The two most frequent malignant brain neoplasms are primary malignant brain tumor and

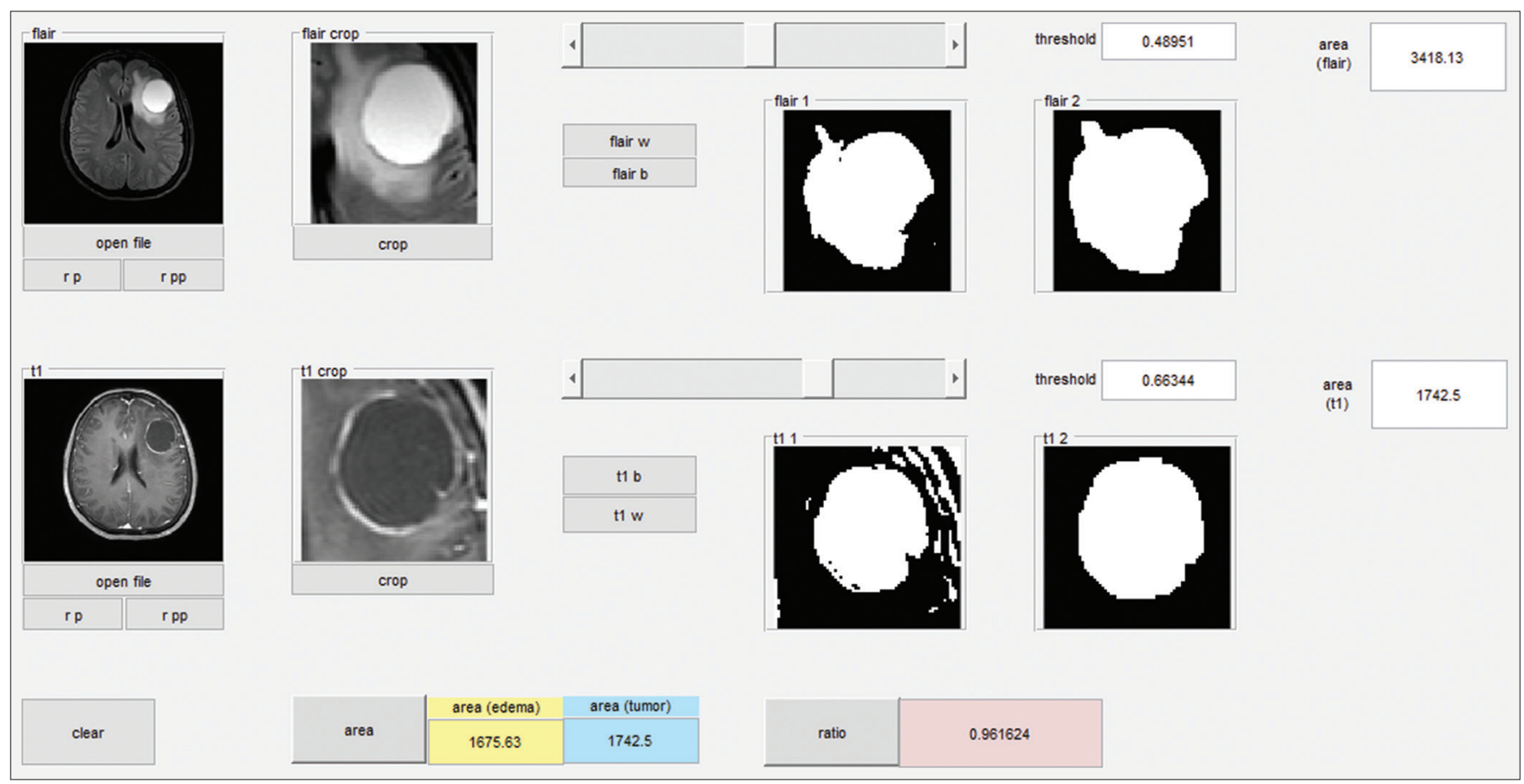

Figure 1: Graphical user interface of the ratio of the area of edema peritumoral to the area of tumor between primary malignant brain tumor and brain metastases 
brain metastases. In a number of cases where patients present with multiple lesions, the diagnosis of brain metastases is usually immediate and not complicated. However, differences may emerge when a patient exhibits a solitary lesion. On displayed by conventional MRI, primary malignant brain tumor and solitary metastatic brain tumor oftentimes exhibit similar contrast enhancement patterns and signal intensity characteristics. Accurately, distinguishing primary malignant brain tumors from brain metastases, and vice versa, becomes crucial due to the different clinical management and treatment of the two.

This study presents the area of peritumoral edema of the two tumor groups having value akin to each other, in which the primary malignant brain tumor group showed a value of 3018.95 (4595.72-183.25), while the metastases group showed a value of 2771.83 (6075.08-236.70). The primary malignant group had a tumor area of 2376.69 (5293.00-211.87), whereas the brain metastases group had a tumor area of 898.12 (4619.75-123.87). If a comparison is made with the data of the primary malignant brain tumor group, then the brain metastases group displays a disproportionate tumor area, where its value was considerably less than its peritumoral edema area. Statistical test results show that there was a significant difference regarding the ratio of the area of peritumoral edema to the area of tumor between the primary malignant brain tumor group and the brain metastases group. Based on the test results of the ratio data between peritumoral edema area and tumor area, this study observed that the primary malignant brain tumor group has a median value $\left(\mathrm{N}_{\max }-\mathrm{N}_{\min }\right)$ of 1.14 (3.31-0.08), while the brain metastases group has a median value $\left(\mathrm{N}_{\max }-\mathrm{N}_{\min }\right)$ of 1.17 (10.30-0.90). The primary malignant brain tumor group had a lower ratio than the brain metastases group. This is in accordance with the hypothesis stating that, in the cases of brain metastases, there is a significant increase in the ratio of vasogenic edema to the increase in lesion size, thus bigger edema when compared to a primary malignant brain tumor.

Primary brain tumor originates from the mutation of neuron cells or glial cells in the brain tissue, which leads to rapid cell division, causing hypercellularity. Because the accelerated mass growth requires angiogenesis to support its growth, cases of primary brain tumors also show increased activity of vascular endothelial growth factor (VEGF). Increased VEGF activity may lead to the disruption of the bloodbrain barrier (BBB). BBB disruption leads to plasma fluid leakage in the brain capillaries and, in turn, results in vasogenic edema in the peritumoral area. Primary brain tumor has a smaller peritumoral edema area than brain metastases because of the presence of hypercellularity within the intracellular space that causes the formation of masses with peritumoral diffuse infiltration, thus allowing the fluid level in the extracellular space to decrease.
Brain metastases arise from the spread of cancer cells from organs other than the brain. This expansion commonly takes place through the bloodstream, though it can also happen through direct geographical invasion, perineural extension, or perivascular extension. The cancer clonal cells that have spread then develop, forming localized mass on the brain intraparenchymal area. Brain metastases also exhibit a high VEGF, which immensely aids the increase in vascular permeability. The increased activity of VEGF can disrupt the BBB. This disruption also induces plasma fluid leakage in the brain capillaries and forms vasogenic edema in the peritumoral area. Brain metastases have a bigger area of peritumoral vasogenic edema than primary malignant brain tumor because of the absence of tumor infiltration, thus resulting in possible excess fluid levels in the extracellular space.

This application of the segmentation method for digital image processing provides additional assistance for diagnosis to differentiate primary malignant brain tumor cases and brain metastases cases through conventional MRI sequences. This technology can be employed as a second opinion for quick screening and early detection in the classification of brain tumors due to the automatic process. Bearing in mind that this technology undertakes the assessment quantitatively, the diagnosis process will become more objective during the classification process. Eventually, the application of this technology may improve the physician's confidence and the patient's trust. Therefore, the quality of service will see improvements due to providing more optimal treatment for both primary malignant brain tumor patients and brain metastases patients using the aforementioned conventional MRI technology.

\section{Conclusions}

This study presents the result illustrating significant difference regarding the ratio of the area of peritumoral edema to the area of tumor between the patient group with a primary malignant brain tumor and the patient group with brain metastases, in which the primary malignant brain tumor group has a lower ratio than the brain metastases group. A GUI designed using the digital image processing method can allow for an easier and more rapid calculation process for the area of tumor and edema with a more precise value result.

\section{References}

1. Simamora SK, Zanariah Z. Space occupying lesion (SOL). J Medula. 2017;7(1):69-73. 
2. Cramer JK, Gerstner ER, Emblem KE, Andronesi O, Rosen B. Advanced magnetic resonance imaging of the physical processes in human glioblastoma. Cancer Res. 2014;74(17):4622-37. https://doi.org/10.1158/0008-5472.CAN-14-0383

PMid:25183787

3. Meyer VJE, Mabray MC, Cha S. Current clinical brain tumor imaging. Clin Neurosurg. 2017;81(3):397-415. https://doi. org/10.1093/neuros/nyx103

PMid:28486641

4. Rahmathulla G, Toms SA, Weil RJ. The molecular biology of Brain metastases. J Oncol. 2012;2012:723541. https://doi. org/10.1155/2012/723541

PMid:22481931

5. Mabray MC, Barajas RF, Cha S. Modern brain tumor imaging Brain Tumor Res Treat. 2015;3(1):8-23. https://doi.org/10.14791/ btrt.2015.3.1.8

PMid:25977902

6. Wang S, Kim S, Chawla S, Wolf RL, Knipp DE, Vossough A et al. Differentiation between glioblastomas, solitary brain metastases, and primary cerebral lymphomas using diffusion tensor and dynamic susceptibility contrast-enhanced MR imaging. Am J Neuroradiol. 2011;32(3):507-14. https://doi. org/10.3174/ajnr.A2333

PMid:21330399

7. Amborsini RD, Wang P, O'Dell WG. Computer-aided detection of metastatic brain tumors using automated 3-D template matching. J Magn Reson Imaging. 2010;31(1):85-93. https:// doi.org/10.1002/jmri.22009

PMid:20027576

8. Owonikoko TK., Arbiser J, Zelnak A, Shu HG, Shim H, Robin AM, et al. Current approaches to the treatment of metastatic brain tumours. NIH Public Access. 2014;11(4):203-22. https://doi. org/10.1038/nrclinonc.2014.25

PMid:24569448

9. Pope WB. Brain metastases: Neuroimaging. Handb Clin Neurol. 2018;149:89-112. https://doi.org/10.1016/ B978-0-12-811161-1.00007-4

PMid:29307364

10. Kunimatsu A, Kunimatsu N, Kamiya K, Watadani T, Mori H, Abe O. Comparison between glioblastoma and primary central nervous system lymphoma using MR image-based texture analysis. Magn Reson Med Sci. 2017;17(1):50-7. https://doi. org/10.2463/mrms.mp.2017-0044 PMid:28638001

11. Arya RS, Ashok K. Texture, shape and color based classification of satellite images using GLCM and Gabor filter, Fuzzy C Means and Svm. Int Res J Eng Technol. 2018;5(4):751-4.

12. Ambarwati $A$, Passarella $R$, Sutarno. Segmentasi Citra Digital Menggunakan Thresholding Otsu untuk Analisa Perbandingan Deteksi Tepi. Vol. 2. Annual Research Seminar; 2016. p. 216-26.

13. Passiglia F, Caglevic C, Giovannetti E, Pinto JA, Manca P, Taverna S, et al. Primary and metastatic brain cancer genomics and emerging biomarkers for immunomodulatory cancer treatment. Semin Cancer Biol. 2018;52(Pt 2):259-68. https://doi. org/10.1016/j.semcancer.2018.01.015

PMid:29391205

14. Hanum $A$, Aslam $A B$, Yueniwati $Y$, Retnani DP, Setjowati $N$. Measurement of the peritumoral edema and tumor volume ratio in differentiating malignant primary and metastatic brain tumor. GSC Biol Pharm Sci. 2020;13(2):55-61. https://doi. org/10.30574/gscbps.2020.13.2.0295

15. Zhou H, Wu J, Zhang J. Digital Image Processing: Part 1. London: Ventus Publishing; 2010. 